\title{
Microring Distributed Sensors Using Electron Cloud Generated by Four-point Probe Sagnac Interferometer
}

\author{
Anita Garhwal 1, Arumona Edward Arumona 2,3,4, Kanad Ray 5, Phichai Youplao ${ }^{6}$, Ghanshyam Singh 7 and \\ Preecha Yupapin 2,3,* \\ 1 Amity School of Engineering and Technology, Amity University Rajasthan, Jaipur, India. \\ 2 Computational Optics Research Group, Advanced Institute of Materials Science, Ton Duc Thang University, \\ Ho Chi Minh City, Vietnam. \\ 3 Faculty of Applied Sciences, Ton Duc Thang University, Ho Chi Minh City, Vietnam. \\ 4 Division of Computational Physics, Institute for Computational Science, Ton Duc Thang University, Ho Chi \\ Minh City, Vietnam. \\ 5 Amity School of Applied Sciences, Amity University Rajasthan, Jaipur, India. \\ 6 Department of Electrical Engineering, Faculty of Industry and Technology, Rajamangala University of Tech- \\ nology Isan Sakon Nakhon Campus, Sakon Nakhon, Thailand. \\ 7 Department of ECE, Malaviya National Institute of Technology Jaipur (MNIT) Jaipur, India. \\ * Correspondence: preecha.yupapin@tdtu.edu.vn
}

\begin{abstract}
A micro Sagnac interferometer integration is proposed for electron cloud distributed sensors. The Sagnac interferometer consists of four microring probes integrated into a Sagnac loop. Each of the microring probes is embedded with the silver bars to form the plasmonic wave oscillation. At the center microrings, electrons are trapped and oscillated by the whispering gallery modes (WGMs), where the plasmonic antennas are established and applied for wireless fidelity (WiFi) and light fidelity $(\mathrm{LiFi})$ transmissions for distributed sensors. The antenna gains are $2.59 \mathrm{~dB}, 0.93 \mathrm{~dB}$, $1.75 \mathrm{~dB}$, and $1.16 \mathrm{~dB}$ respectively for the four antennas formed at the center microrings. The polarized light of $1.50 \mu \mathrm{m}$ wavelength is fed into the interferometer input, which is polarized randomly into upstream and downstream directions. The polarization components can be obtained by the spacetime modulation control. By controlling the electron cloud spin orientation, the space-time projection can be applied, and the ultra-high measurement resolution can be obtained in terms of fast switching time (change in phase). In manipulation, the applied stimuli are substituted by the change in input source power. The light input power variation causes a change in electron cloud density. Similarly, when the electron cloud is excited by the microscopic medium, which can be employed as the microscopic sensors. The WGM sensors have sensitivities of $1.35 \mu \mathrm{m}^{-2}, 0.90 \mu \mathrm{m}^{-2}, 0.97 \mu \mathrm{m}^{-2}$ and, $0.81 \mu^{-2}$, respectively. The WGMs behave as a four-point probe for the electron cloud distributed sensors, where the electron cloud sensitivities of 2.31 prads $^{-1} \mathrm{~mm}^{3}$ (electrons) ${ }^{-1}, 2.27 \mathrm{prads}^{-1} \mathrm{~mm}^{3}$ (electrons) $)^{-1}, 2.22$ prads $^{-1} \mathrm{~mm}^{3}$ (electrons) ${ }^{-1}, 2.38$ prads $^{-1} \mathrm{~mm}^{3}$ (electrons) ${ }^{-1}$ are respectively obtained.
\end{abstract}

Keywords: distributed sensors; Sagnac interferometer; microring sensors; electron cloud sensors

\section{Introduction}

An interferometer is an optical device which is employed as sensors for many physical quantities. An interferometer has different configurations which are employed for sensing applications [1-8]. The Sagnac interferometer is one of the configurations of an interferometer which is the focus of this work. The Sagnac interferometer setup usually involves a ring configuration where the light beam splits into two beams. The two beams travel around the ring path but in opposite directions and the two beams undergo interference on returning to the entry point of the Sagnac interferometer. There are different types of Sagnac interferometers that have been designed, developed, and employed for sensing applications. Liu et al. [9] designed a Sagnac interferometer that consists of circular photonic crystal fiber. The Sagnac interferometer is employed for temperature sensing. 
The finite element method is employed in the simulation. The average sensitivity of $18.27 \mathrm{~nm}^{\circ} \mathrm{C}^{-1}$ is obtained. The wavelength range of $0.5-2.5 \mu \mathrm{m}$ is employed in the study. Liu et al. [10] designed and fabricated a Sagnac interferometer that consists of polarized photonic crystal fiber. The Sagnac interferometer is employed for force sensing. The force sensor in the study is insensitive to temperature. In the experimental study, the wavelength range of $1510-1630 \mathrm{~nm}$ is employed. The sensitivities of $8.16 \mathrm{nmN}^{-1}, 11.22 \mathrm{nmN}^{-1}$, and $16.32 \mathrm{nmN}^{-1}$ are obtained respectively when the force is varied from 0 to $0.392 \mathrm{~N}$. Lv et al. [11] designed and fabricated a Sagnac interferometer that consists of microfiber of singlemode and ferrofluid. The Sagnac interferometer is employed for a magnetic field sensor. The wavelength range of $1530-1535 \mathrm{~nm}$ is employed for the study. The sensitivity of $0.5 \mathrm{pm}(\mathrm{Oe})^{-1}$ is obtained. Mollah et al. [12] designed a Sagnac interferometer that consists of photonic crystal fiber. The Sagnac interferometer is employed for salinity sensing. The finite element method is employed in the simulation. The wavelength range of $1-2.5 \mu \mathrm{m}$ is

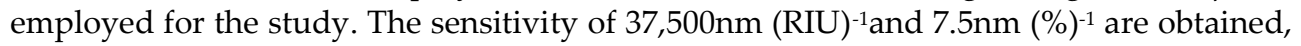
respectively. Chen et al. [13] designed a Sagnac interferometer that consists of side-leakage photonic crystal fiber. The Sagnac interferometer is employed for torsion sensing. In the experimental study, the wavelength range of $1400 \mathrm{~nm}-1750 \mathrm{~nm}$ is employed. The sensitivity of $0.935 \mathrm{~nm}\left({ }^{\circ}\right)^{-1}$ is obtained. Zu et al. [14] designed and developed a Sagnac interferometer that consists of low birefringence photonic crystal fiber. The Sagnac interferometer is employed for a twist sensor. In the experimental study, the wavelength range of $1520-1620 \mathrm{~nm}$ is employed. The sensitivity of $1.00 \mathrm{~nm}\left({ }^{0}\right)^{-1}$ is obtained. Gong et al. [15] designed a Sagnac interferometer that consists of hollow-core photonic crystal fiber. The Sagnac interferometer is employed for curvature sensing. In the experimental study, the wavelength range of $1520-1580 \mathrm{~nm}$ is employed. In the curvature range of $0-9.9 \mathrm{~m}^{-1}$ the sensitivity of $0.232 \mathrm{~nm}(\mathrm{~m})$ is obtained. Feng et al. [16] designed two Sagnac interferometers that consist of polarized and suspended core (SC) polarized photonic crystal fibers. The two Saganc interferometers are employed for pore water pressure sensing. In the experimental study, the wavelength range of $1510 \mathrm{~nm}-1590 \mathrm{~nm}$ is employed. The SC polarized Sagnac interferometer has an average sensitivity of $254.75 \mathrm{kpa}(\mathrm{nm})^{-1}$ while the polarized Sagnac interferometer has an average sensitivity of $304.41 \mathrm{kpa}(\mathrm{nm})^{-1}$. Li et al. [7] designed a Sagnac interferometer that consists of exposed-core fiber. The Sagnac interferometer is employed for bio-sensing. In the experimental study, the wavelength range of 1200nm$1450 \mathrm{~nm}$ is employed. The biosensor in the study has a sensitivity of $-3,137 \mathrm{~nm}$ (RIU)-1. Sun et al. [17] designed and fabricated a Sagnac interferometer that consists of a birefringence elliptical microfiber. The Sagnac interferometer is employed for relative humidity (RH) sensing. In the experimental study, the wavelength range of $1450 \mathrm{~nm}-1650 \mathrm{~nm}$ is employed. The sensitivity of $\sim 201.25 \mathrm{pm}(\% \mathrm{RH})^{-1}$ is obtained in the $\mathrm{RH}$ range of $30 \% \mathrm{RH}$ to $90 \% \mathrm{RH}$. The study employed a panda fiber for the Sagnac interferometer and the sensitivity of $\sim 422.2 \mathrm{pm}(\% \mathrm{RH})^{-1}$ is obtained thereby enhancing the sensitivity. An et al. [18] designed a Sagnac interferometer that consists of photonic crystal fiber. The Sagnac interferometer is employed for glucose sensing. The finite element method is employed in the simulation. The average sensitivity of $22,130 \mathrm{~nm}(\mathrm{RIU})^{-1}$ is obtained. Wu et al. [19] designed a Sagnac interferometer that consists of polarized optical fiber. The Sagnac interferometer is based on the vernier effect. The Sagnac interferometer is employed for hydrogen sensing. In the experimental study, the wavelength range of $1460 \mathrm{~nm}-1600 \mathrm{~nm}$ is employed. The sensitivity of $-14.61 \mathrm{~nm}(\%)^{-1}$ is obtained in the range of $0 \%-0.8 \%$. In this work, an integrated Sagnac interferometer is employed for electron cloud sensors. Four microrings are embedded with the silver bars at the center of the microrings, from which the electro-optic sensing probes can be formed. An isolator is used at the input to control the reflected signal. Four whispering gallery modes (WGMs) are formed by the nonlinear effect coupled by the two side rings, from which the electron cloud is trapped and oscillated by the plasmonic waves. These WGMs act as four-probe sensors. The polarized laser is fed into the Sagnac interferometer, from which the output is split by a beamsplitter. The OptiFDTD software 
is employed for the design and simulation while the Matlab program employs the extracted parameters from the simulation results to plot the graphs and obtain other results.

\section{Theoretical Background}

The Sagnac interferometer circuit is shown in Figure 1. The input signal is the polarized wave [20-21], which is given by an equation (1).

$$
E_{i n}(t)=D \exp ^{-\alpha L+j \varphi_{0}(t)}
$$

where $D$ is the amplitude of the optical field, and $L$ is the waveguide length. $\alpha$ and $\varphi_{0}$ are the attenuation and phase constants.

The propagation of light pulse within the nonlinear material and in refractive index $(n)$ is given as [20-21].

$$
n=n_{0}+n_{2} I=n_{0}+\frac{n_{2}}{A_{\text {eff }}} P
$$

where $n_{0}, n_{2}, I$ and $P, A_{\text {eff }}$ are the linear and nonlinear refractive indices, optical intensity and optical power, effective core area, respectively. The light pulse follows the two paths for propagation which are clockwise and anticlockwise. After traveling through two paths, a phase shift is introduced in the output pulse.

The electron behavior of the silver bars at the four center microrings is described by the Drude model [22], which is given as:

$$
\epsilon(\omega)=1-\frac{n_{e} e^{2}}{\epsilon_{0} m \omega^{2}}
$$

where $n_{e}$ is the electron density, $m$ is the mass, $\omega$ is the angular frequency, $\epsilon_{0}$ is the relative permittivity, $e$ is the electron charge. The plasma frequency $\left(\omega_{p}\right)$ at the resonance is obtained from the angular frequency, which is given by the equation (4) [22].

$$
\omega_{p}=\left[\frac{n_{e} e^{2}}{\epsilon_{0} m}\right]^{-1 / 2}
$$

from equation (4), $n_{e}=\frac{\omega_{p \epsilon_{0} m}^{2}}{e^{2}}$.

The space time modulation signal is applied at the add port $\left(E_{\text {add }}\right)$ of the microring, the polarization component can be calculated and used for quantum interpretation. The space time signal is given by an equation (5) [23].

$$
E_{\text {add }}=A e^{ \pm i \omega t}
$$

where A, $\omega$, and $t$ are amplitude, angular frequency, and time. A space time modulation signal is applied to achieve polarized output. The \pm shows both axes of time.

The excited electrons are trapped inside the four microring resonators, where the four of WGMs' formations are taken place. The output at throughput $\left(E_{t h}\right)$ and drop ports $\left(E_{d r}\right)$ are given by [22-23].

$$
\begin{aligned}
& E_{t h}=m 2 E_{\text {in }}+m 3 E_{a d d} \\
& E_{d r}=m 5 E_{a d d}+m 6 E_{\text {in }}
\end{aligned}
$$

where $m 2, m 3, m 5$, and $m 6$ are constants. The system outputs are normalized intensities, which are given as:

$$
\begin{aligned}
& \frac{I_{t h}}{I_{\text {in }}}=\left[\frac{E_{t h}}{E_{\text {in }}}\right]^{2} \\
& \frac{I_{d r}}{I_{\text {in }}}=\left[\frac{E_{d r}}{E_{\text {in }}}\right]^{2}
\end{aligned}
$$

where $I_{t h}, I_{i n}$, and $I_{d r}$ are the throughput, input and drop port intensities, respectively. The phase shift is given in equation (10) [24]. 


$$
\phi=\pi-\frac{\beta L}{2}-\arctan \left(\frac{\kappa_{2} \kappa_{4} \gamma_{1} \gamma_{3} \alpha^{4} \sin \beta L}{1-\kappa_{2} \kappa_{4} \gamma_{1} \gamma_{3} \alpha^{4} \cos \beta L}\right)
$$

where $\kappa_{2}, \kappa_{4}$ are coupling coefficients, $\gamma$ is insertion loss, $\alpha$ is attenuation coefficient, $\beta$ is propagation constant and $L=2 \pi r$ where $r$ is the radius of the center microring. The integrated system for the microring distributed electron cloud sensor network is designed and simulated in OptiFDTD. The four microring circuits inside the Sagnac form the WGM. The designed system is explained using equations (1) to (10).
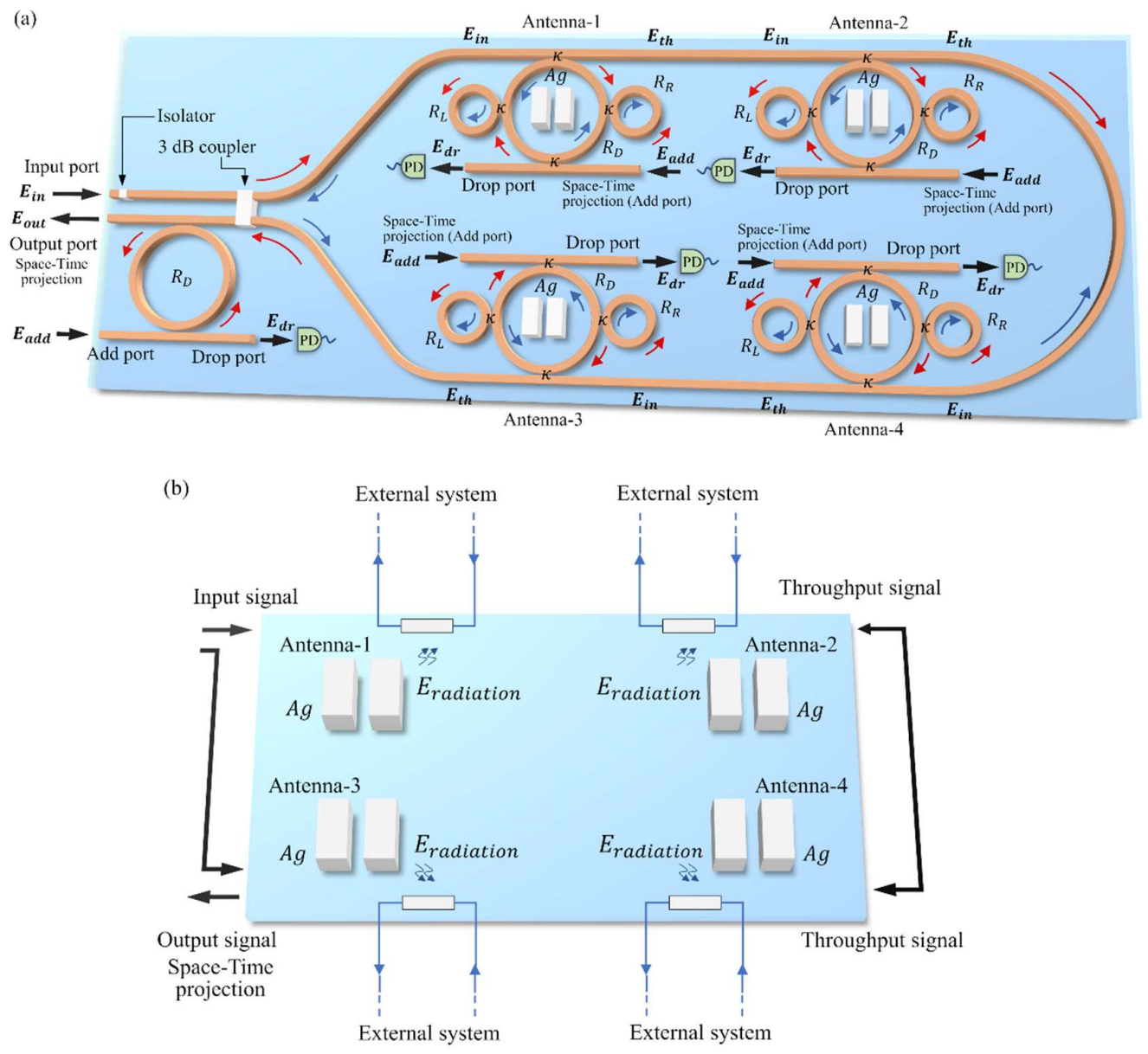

Figure 1. The device fabricated structure of proposed work, where (a) micro-electron cloud sensors network system; Ein, Eth, Edr, E add are electric fields at input, throughput, drop and add ports, respectively. $\kappa$ is coupling constants. PD is a beam splitter which gives the polarized laser components, (b) a sensing probe circuit. The micro rings are embedded with silver (Ag) nano bars. The optical isolator is applied to project the feedback to the laser source.

\section{Methods}

Figure 1 shows the designed structure of the microring distributed electron cloud sensors system. The input signal is applied at the input port. The input light is a polarized laser. The materials of Sagnac and microring are silica and Si, respectively. The light travels in the upper and lower branches of the Sagnac loop. The Sagnac loop is embedded with four microring resonator circuits. The radius of center microring $\left(R_{D}\right)$ is bigger than the side ring radius $\left(R_{L}, R_{R}\right)$. The design parameters of the proposed system are shown in Table 1 . The applied microring circuit ports depend on the light flow direction in the 
Sagnac loop, which is the two-way propagation, where the Sagnac closed loop is the required result. The system is designed and simulated in OptiFDTD for 20,000 time-steps. The data is extracted from OptiFDTD, and graphs are plotted using MATLAB software, where Figures 2-7 show the results of the designed system. The working of the designed system is as follows. First, the input signal of wavelength $1.50 \mu \mathrm{m}$ is applied at the input port, which is a polarized laser as shown in an equation (1). Half-light energy propagates into the upper branch (upstream), and the other half into the lower branch (downstream) directions, respectively. The signal flow of the upper branch is in the clockwise direction. The lower branch is the anticlockwise direction, which is shown in Figure 1 where four microrings are integrated with the Sagnac loop.
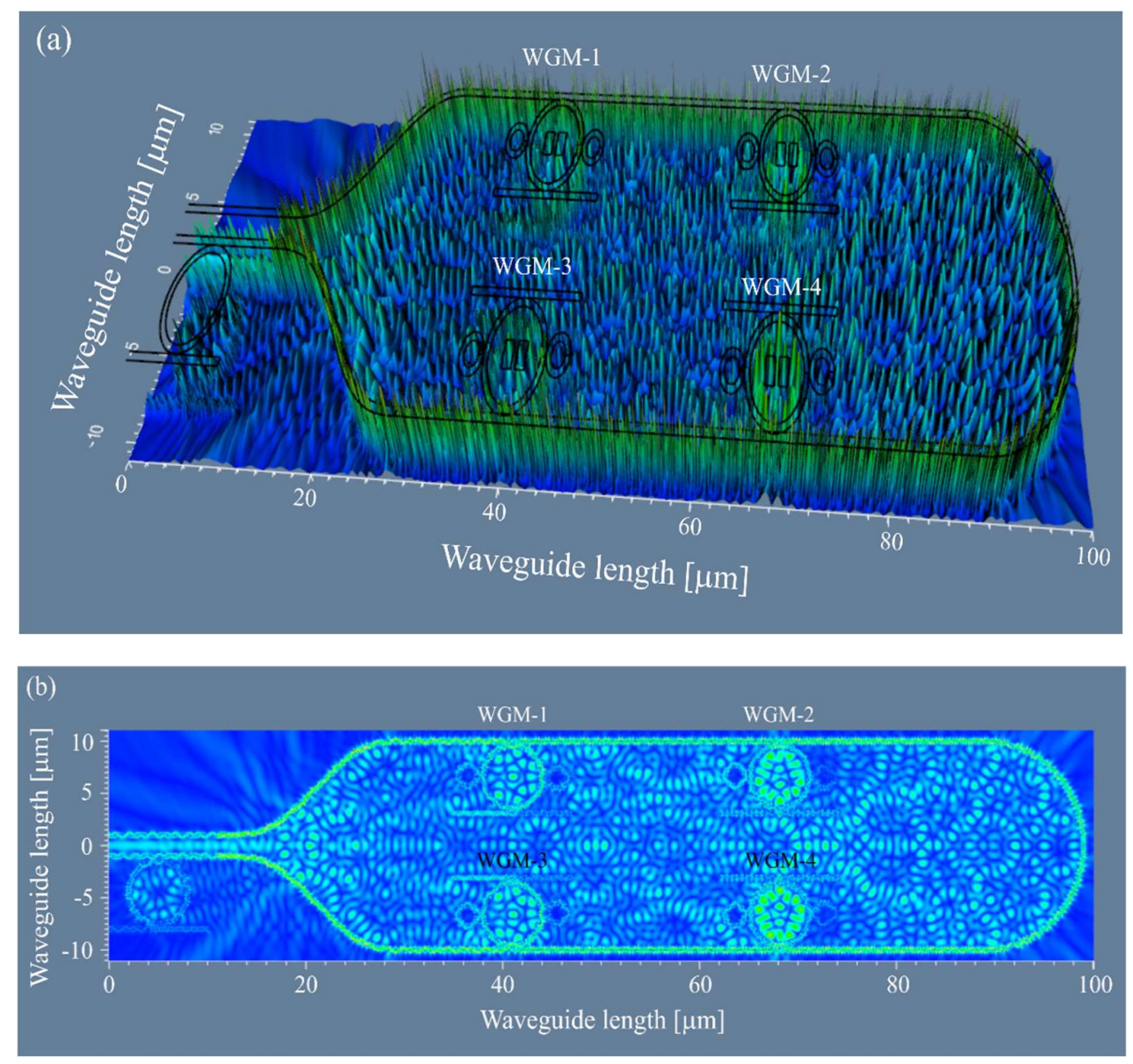

Figure 2. The Optiwave graphical results, where (a) WGMs formation; (b) plasmons propagating in the system. Input source is a polarized laser with of wavelength $1.50 \mu \mathrm{m}$. The used parameters of simulation are given in Table 1 .

The two rings are with the upper branch and the other two with the lower branch. The micro rings are embedded with silver nano bars. Light propagates into the input port of microring-1(antenna-1), from the throughput port of antenna-1 enters into the input port of microring-2(antenna-2). It is the same operation with antenna-3 and 4. Light from the antenna- 2 and 4 throughput ports enter the throughput ports of 4 and 2, which become the input of the closed-loop operation. Similarly, from the throughput signals of antenna4 enters the input port of antenna- 3 and from the throughput of antenna-2 to antenna-1. Both paths of propagated lights return at the $3 \mathrm{~dB}$ coupler. The optical isolator is applied at the input port to prevent the reflection of the backlight. The light goes into outer microring at the output port. The propagation of the light pulse in the nonlinear material is 
given by equation (2). The real-time experiment of the Sagnac interferometer consists of a beam-splitter. Here, the proposed system is manipulated using space-time modulation at the add port of the microrings. The Sagnac output enters into the microring, which is polarized light. The light propagates inside the microring circuits, from which the phase shift between the polarization components is obtained by the space-time projection control. The Kerr effect is a nonlinear effect induced by the system, and with suitable parameters, as shown in Table 1, where the WGMs are formed. The WGM is the trapping of light at the center microring. These WGMs form the antennas inside the Sagnac which consist of the trapped electrons resulting from the illumination of the silver nano-bars by light at the center microring. Figure 2 shows the graphical results of the simulated structure.

Table 1. The parameters used in simulation parameters.

\begin{tabular}{|c|c|}
\hline Parameters & Values \\
\hline Input power $(\boldsymbol{P})$ & $10-15 \mathrm{~mW}$ \\
\hline Input wavelength $(\boldsymbol{\lambda})$ & $1.50 \mu \mathrm{m}$ \\
\hline Center ring radius $\left(\boldsymbol{R}_{\boldsymbol{D}}\right)$ & $2.0 \mu \mathrm{m}$ \\
\hline Side rings radius $\left(\boldsymbol{R}_{\boldsymbol{L}}, \boldsymbol{R}_{\boldsymbol{R}}\right)$ & $0.80 \mu \mathrm{m}$ \\
\hline Silver refractive index $\left(\boldsymbol{n}_{\boldsymbol{A g}}\right)[25]$ & 0.14 \\
\hline Coupling coefficient $(\boldsymbol{\kappa})[23]$ & 0.50 \\
\hline Insertion loss $(\boldsymbol{\gamma})$ & $0.50 \mathrm{~dB}$ \\
\hline Structure dimensions $(\boldsymbol{L} \boldsymbol{x} \boldsymbol{W})$ & $100 \times 20 \mu \mathrm{m}^{2}$ \\
\hline Silver bar length $(\boldsymbol{l})$ & $1.25 \mu \mathrm{m}$ \\
\hline Silver bar width $(\boldsymbol{w})$ & $2.0 \mu \mathrm{m}$ \\
\hline Silica refractive index $\left(\boldsymbol{n}_{\boldsymbol{s i o}}\right)$ & 1.45 \\
\hline Silica nonlinear refractive index $\left(\boldsymbol{n}_{\mathbf{2}}\right)$ & $2.7 \times 10^{-16} \mathrm{~m}^{2} \mathrm{~W}^{-1}$ \\
\hline Si- refractive index $\left(\boldsymbol{n}_{\boldsymbol{s i}}\right)$ & 3.42 \\
\hline Fiber loss & $0.1 \mathrm{~dB} \mathrm{~km}^{-1}$ \\
\hline Core effective area $\left(\boldsymbol{A}_{\boldsymbol{e f f}}\right)$ & $0.25 \mu \mathrm{m}^{2}$ \\
\hline Free space permittivity $\left(\boldsymbol{\varepsilon}_{\mathbf{0}}\right)$ & $8.85 \times 10^{-12} \mathrm{Fm}^{-1}$ \\
\hline Electron mass $(\boldsymbol{m})$ & $9.11 \times 10^{-31} \mathrm{~kg}^{-1}$ \\
\hline Electron charge $(\boldsymbol{q})$ & $1.60 \times 10^{-19} \mathrm{Coulomb}^{-1}$ \\
\hline Waveguide loss $(\boldsymbol{\alpha})[23]$ & $0.50 \mathrm{~dB} \mathrm{~mm}^{-1}$ \\
\hline
\end{tabular}

\section{Simulation Results}

Figure 2(a) shows the formation of the four WGMs at the center microrings. Figure 2(b) shows the plasmons that propagate through the system with intense electromagnetic field. Figure 3(a) shows the frequency and input intensity plot of the four WGMs. The peak frequencies of WGM-1 to WGM-4 are 208.43THz, 198.18THz, 206.72, and 196.47THz, respectively while Figure $3(\mathrm{~b})$ shows the wavelength and input intensity plots of the four WGMs. The antenna profiles are plotted in Figures (4-6). Figure 4 shows the antenna gain plot. Antenna gain is plotted by varying the input power from $10 \mathrm{~mW}$ to $15 \mathrm{~mW}$. A linear trend of gain is achieved with input power. The gain of antennas 1 to anetnna 4 are $2.59 \mathrm{~dB}$, $0.93 \mathrm{~dB}, 1.75 \mathrm{~dB}$, and $1.16 \mathrm{~dB}$, respectively. Figure 5 shows the antennas' directivities. Equation (5) gives the space-time applied at the add port and equations (6 and 7) give the microring output at throughput and drop ports. The output at the throughput and drop ports are normalized using equations ( 8 and 9$)$. Figure 6(a-b) is the plot of the normalized electron density of antennas 1 to 4 in the frequency domain and wavelength domain. The frequency domain is employed for the WiFi (wireless fidelity) band while the wavelength domain is employed for LiFi (light fidelity) band. Antennas- 1 to 4 are recognized for both 
$\mathrm{WiFi}$ and LiFi transmission. Gain and directivity are calculated using the standard formula $[22,26]$. Figure 7 shows the output intensity plot of the four WGMs. The input power is varied from $10 \mathrm{~mW}-15 \mathrm{~mW}$.A linear trend of the output intensity of the four WGMs is achieved with input power. The sensitivities of $1.35 \mu \mathrm{m}^{-2}, 0.90 \mu \mathrm{m}^{-2}, 0.97 \mu \mathrm{m}^{-2}$ and, $0.81 \mu \mathrm{m}$ 2 are obtained, respectively. The plasma frequency and electron density are related as given in equation (4). In the silver nano-bars, the electron density oscillations depend on the plasma frequency. Figure 8 shows the plot of the plasma frequency and electron density. The sensitivities of 2.31 prads $^{-1} \mathrm{~mm}^{3}$ (electrons) $)^{-1}, 2.27$ prads $^{-1} \mathrm{~mm}^{3}$ (electrons) ${ }^{-1}$, 2.22 prads $^{-1} \mathrm{~mm}^{3}$ (electrons $)^{-1}, 2.38$ prads $^{-1} \mathrm{~mm}^{3}$ (electrons $)^{-1}$ are respectively obtained. Figure $9(\mathrm{a})$ is the plot of the change in electron density (normalized electron density) and input power. The input power is varied where the sensitivities of $0.06 \mathrm{~mW}^{-1}, 0.04 \mathrm{~mW}^{-1}, 0.05 \mathrm{~mW}$ $1,0.03 \mathrm{~mW}^{-1}$ are obtained respectively for the four probes while Figure $9(\mathrm{~b})$ is the plot of the change in electron density (normalized electron density) and the phase shift (as given in equation (10)) where the sensitivities of $0.005\left(^{\circ}\right)^{-1}, 0.003\left({ }^{\circ}\right)^{-1}, 0.004\left({ }^{\circ}\right)^{-1}, 0.002\left(^{\circ}\right)^{-1}$ are obtained respectively for the four probes. These four WGMs behave as a four-point probe for the electron cloud sensors network. The proposed system has applications in spectroscopy and quantum sensing.
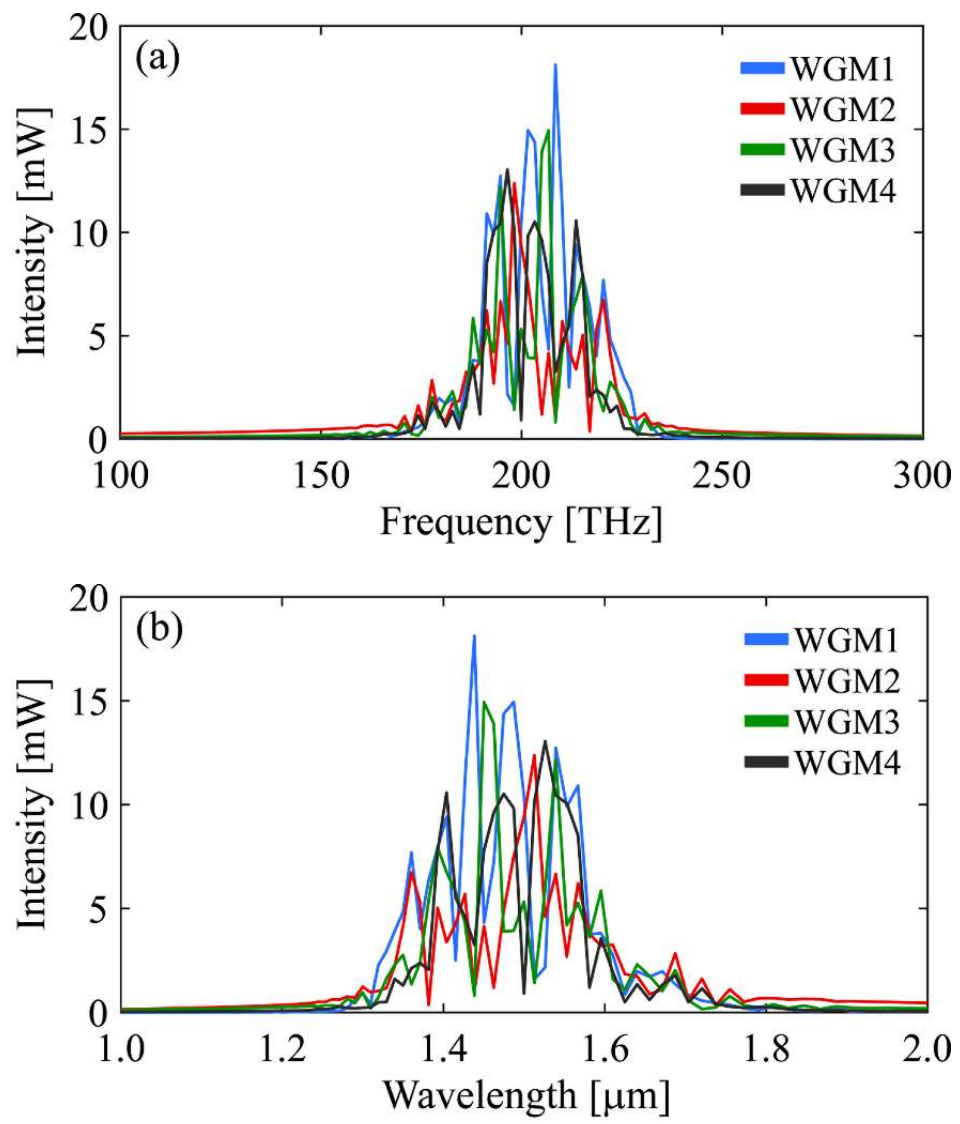

Figure 3. The plot of the input intensities and (a) frequency and (b) wavelength for all WGMs. The peak frequencies of WGM-1, WGM-2, WGM-3, and WGM-4 are 208.43THz, 198.18THz, 206.72 and, 196.47THz, respectively. 


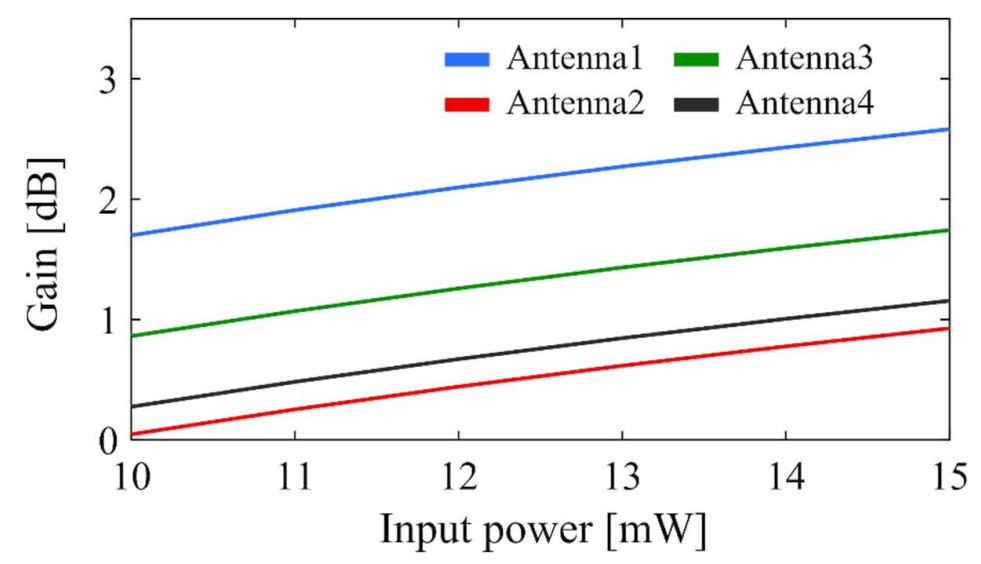

Figure 4. The plot of antennas' gain and input power. The input power varied from $10-15 \mathrm{~mW}$. The obtained gains are $2.59 \mathrm{~dB}, 0.93 \mathrm{~dB}, 1.75 \mathrm{~dB}$, and $1.16 \mathrm{~dB}$ for antenna- 1 , antenna-2, antenna-3, and antenna-4, respectively.
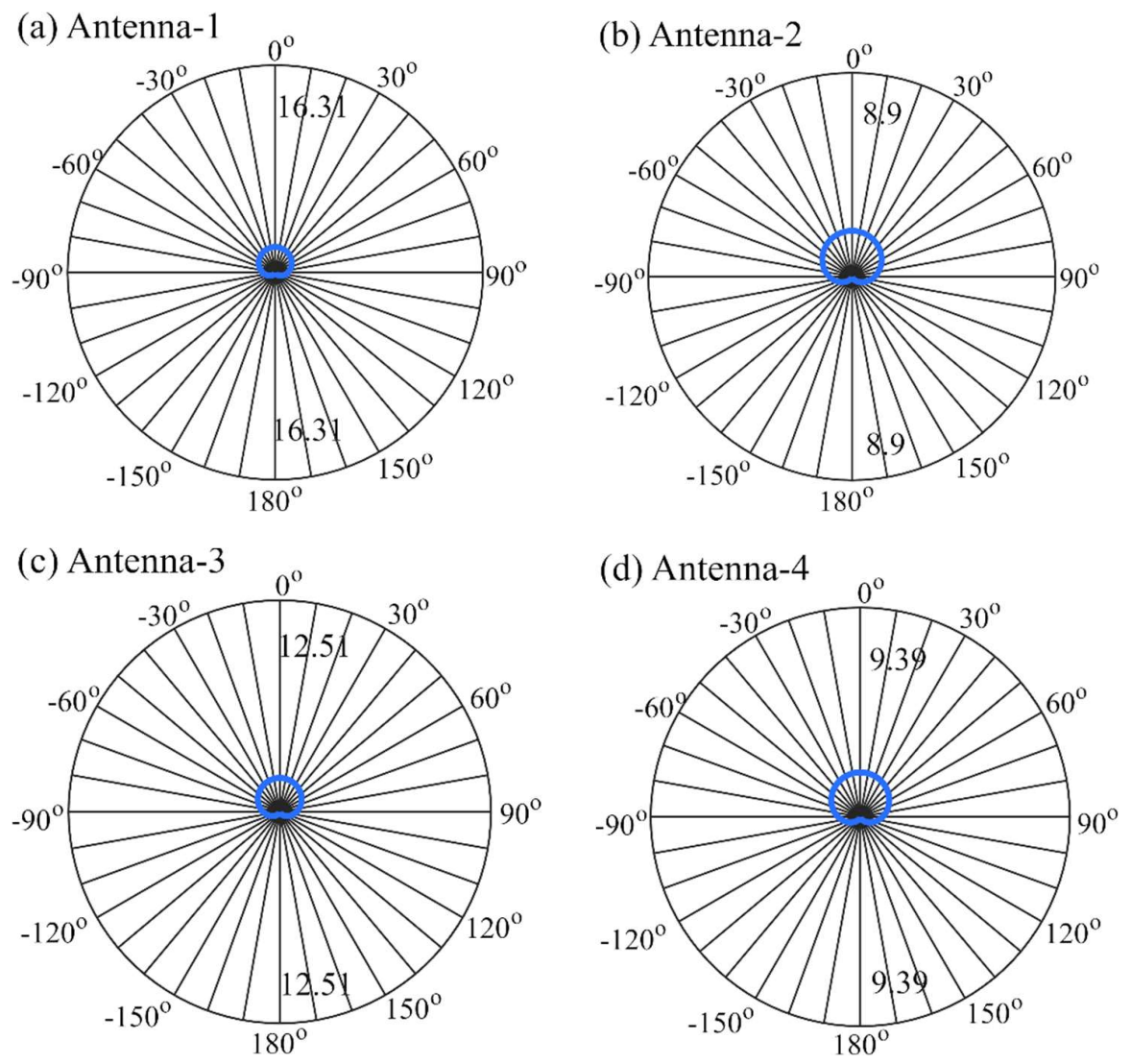

Figure 5. The plot of directivities of antennas, where the obtained directivities are 16.31, 8.9, 12.51, and 9.39 for antenna-1, antenna-2, antenna-3, and antenna-4, respectively. 

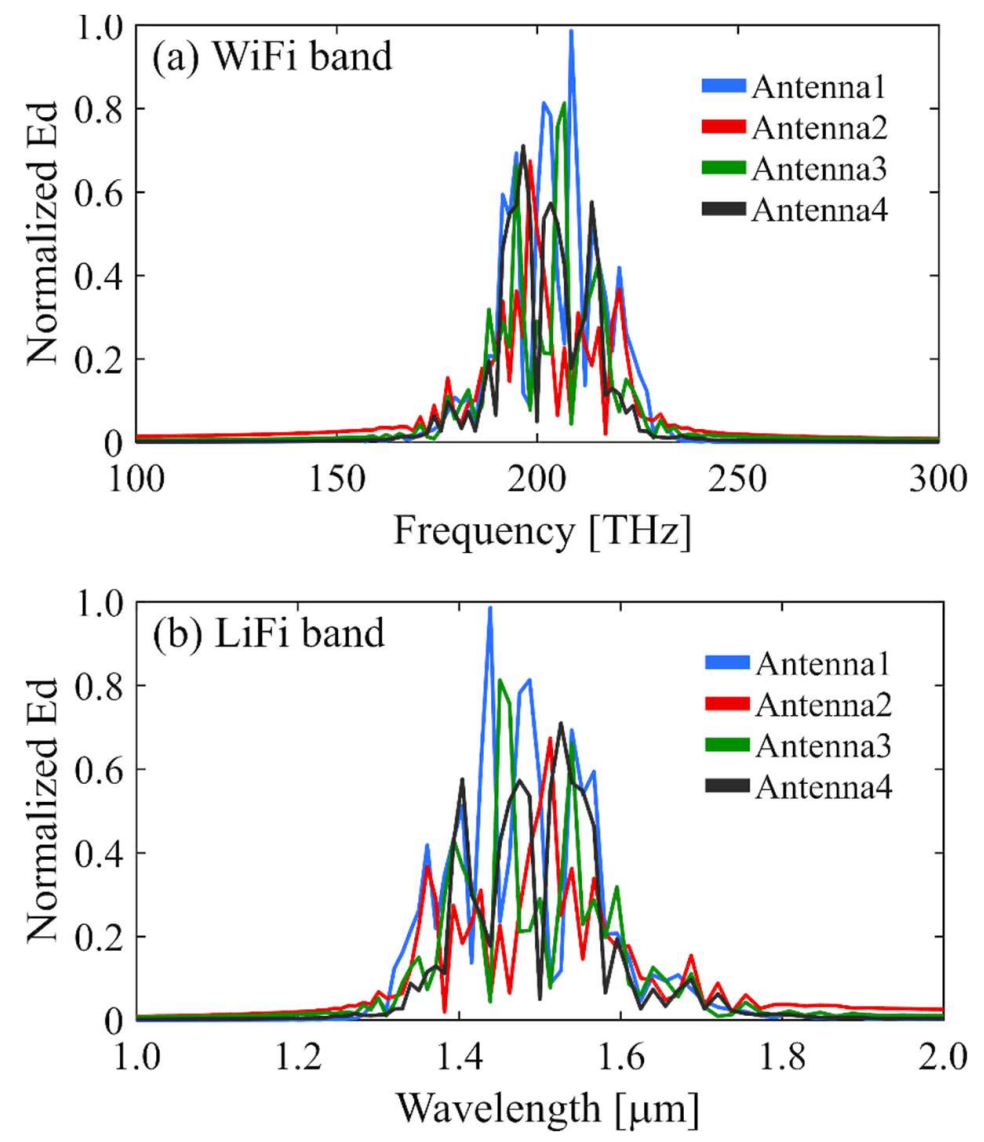

Figure 6. The plot of antenna profiles, where (a)WiFi band frequencies of antenna 1 to 4 are 208.43THz, 198.18THz, 206.72THz, and 196.47THz, respectively, (b) LiFi band wavelengths of antenna 1 to 4 are $1.43 \mu \mathrm{m}, 1.51 \mu \mathrm{m}, 1.45 \mu \mathrm{m}, 1.52 \mu \mathrm{m}$, respectively.

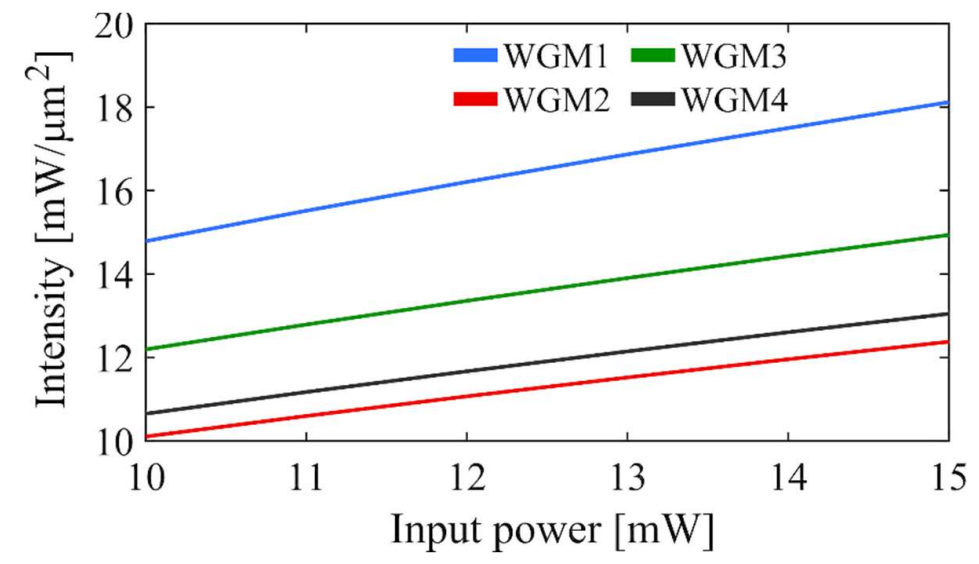

Figure 7. The plot of the output intensities for all WGMs and input power which form the WGM sensors. The input power is varied from $10-15 \mathrm{~mW}$. The sensitivities of $1.35 \mu \mathrm{m}^{-2}, 0.90 \mu \mathrm{m}^{-2}, 0.97 \mu \mathrm{m}^{-2}$, and $0.81 \mu \mathrm{m}^{-2}$, are obtained, respectively. 


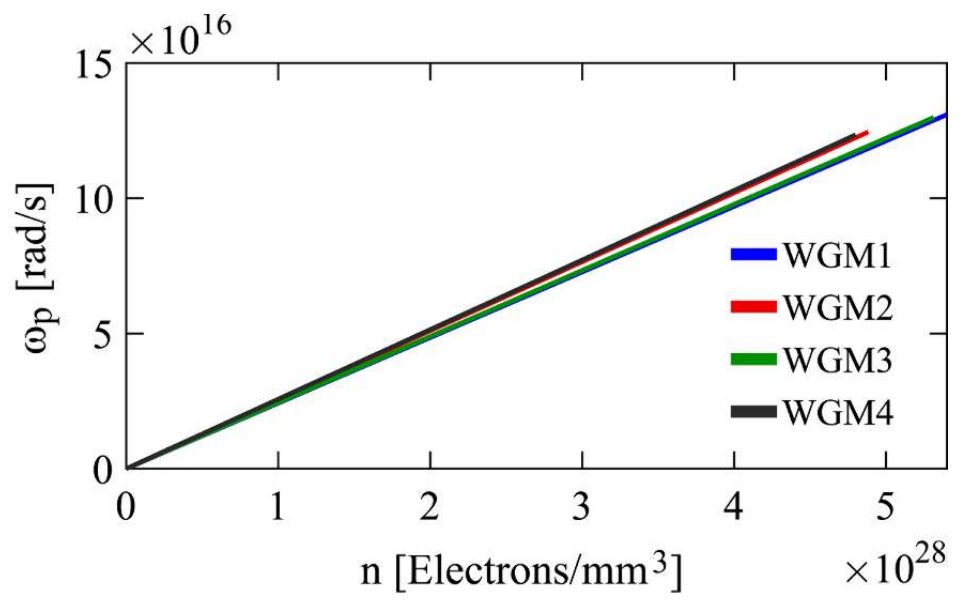

Figure 8. The plot of the plasma frequency and electron density which form the electron cloud sensors. The sensitivities of 2.31 prads $^{-1} \mathrm{~mm}^{3}$ (electrons) ${ }^{-1}, \quad 2.27$ prads $^{-1} \mathrm{~mm}^{3}$ (electrons) ${ }^{-1}$, 2.22prads ${ }^{1} \mathrm{~mm}^{3}$ (electrons) $)^{-1}, 2.38$ prads $^{-1} \mathrm{~mm}^{3}$ (electrons) $)^{-1}$ are respectively obtained.
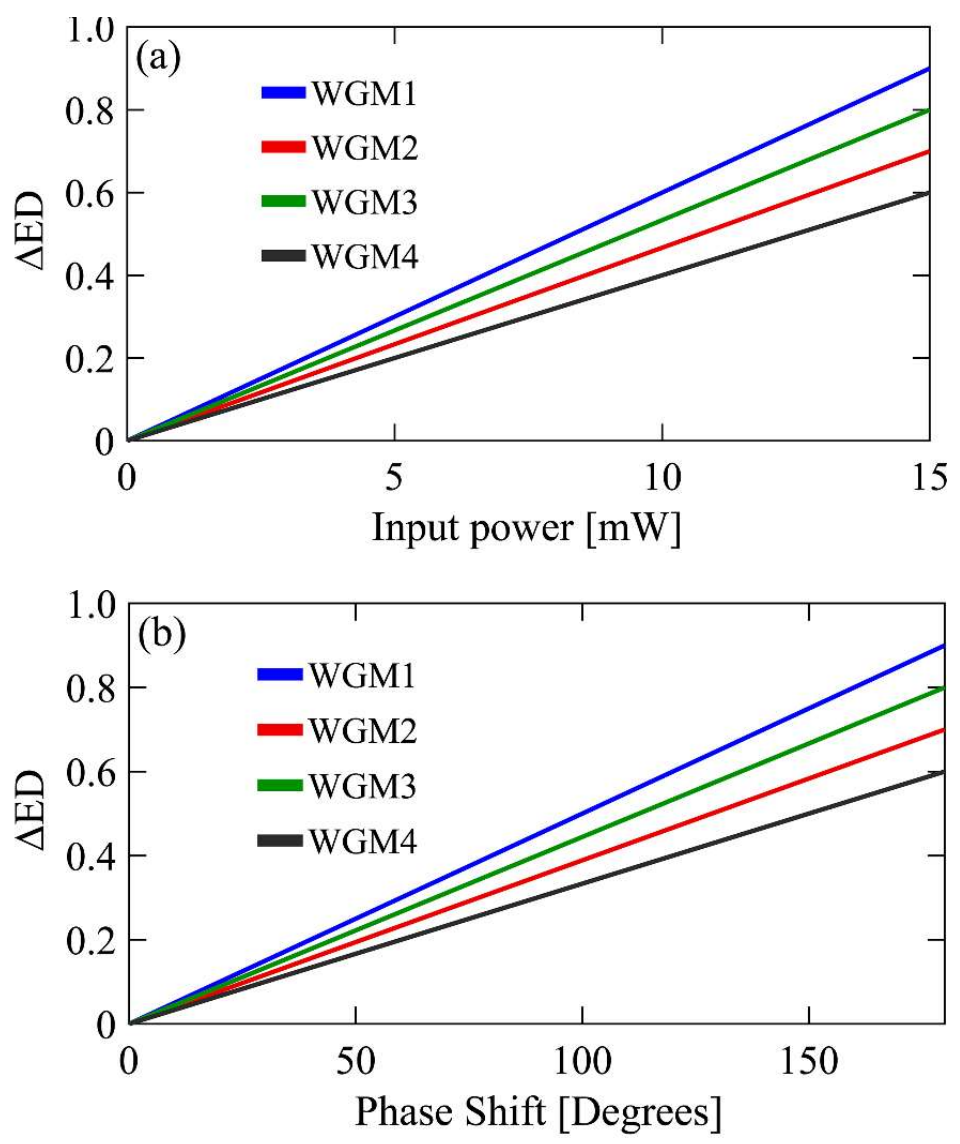

Figure 9. The plot of the (a) change in electron density (normalized electron density) and input power. The sensitivities of $0.06 \mathrm{~mW}^{-1}, 0.04 \mathrm{~mW}^{-1}, 0.05 \mathrm{~mW}^{-1}, 0.03 \mathrm{~mW}^{-1}$ are obtained respectively for WGM1-WGM4, (b) change in electron density (normalized electron density) and phase shift. The sensitivities of $0.005\left({ }^{\circ}\right)^{-1}, 0.003\left({ }^{\circ}\right)^{-1}, 0.004\left({ }^{\circ}\right)^{-1}, 0.002\left({ }^{\circ}\right)^{-1}$ are obtained respectively for WGM1-WGM4. 


\section{Conclusion}

A Sagnac interferometer and microring integrated circuit for electron cloud sensors is proposed. When light propagates inside the Sagnac loop, it goes in both clockwise and anticlockwise directions. The microrings are embedded with silver nano-bars. The electron cloud is trapped inside the microrings, where the WGM formation takes place, the plasmonic wave generates. Four plasmonic antennas formed at the center microrings where the frequency spectrum is employed for WiFi communication and the wavelength spectrum employed for LiFi communication. The obtained antenna gains are $2.59 \mathrm{~dB}$, $0.93 \mathrm{~B}, 1.75 \mathrm{~dB}$, and $1.16 \mathrm{~dB}$ for antenna- 1 to 4 , respectively. These four nodes behave as electron cloud sensors. The WGM output intensity sensor is realized with the sensitivities of $1.35 \mu \mathrm{m}^{-2}, 0.90 \mu \mathrm{m}^{-2}, 0.97 \mu \mathrm{m}^{-2}$, and $0.81 \mu \mathrm{m}^{-2}$ respectively for the four nodes while the electron cloud sensors are realized with the sensitivities of $2.31 \mathrm{prads}^{-1} \mathrm{~mm}^{3}$ (electrons) ${ }^{-1}$, 2.27 prads $^{-1} \mathrm{~mm}^{3}$ (electrons $)^{-1}, 2.22$ prads $^{-1} \mathrm{~mm}^{3}$ (electrons) ${ }^{-1}$, and 2.38 prads $^{-1} \mathrm{~mm}^{3}$ (electrons) $)^{-1}$ respectively for the four nodes. The proposed system has potential applications in quantum sensor and consciousness, from which the electron cloud spins generated by the micro ring antennas can be controlled and analyzed for quantum bits interpretations.

Acknowledgement: The authors would like to acknowledge the research facilities from Ton Duc Thang University, Vietnam.

Funding: Not applicable.

Competing Interests: The authors have declared no conflict of interest.

Availability of data and materials: Not applicable.

Code availability: Not applicable.

Authors Contributions: Anita Garhwal: simulation, analysis, writing original draft, Aumona Edward Arumona: Matlab results improvement, review and discussion, Kanad Ray: modeling, analysis, discussion, final editing, Phichai Youplao: validation, comparing Optiwave and Matlab results, visualization, and discussion, Ghanshyam Singh: improve the writing draft, and discussion, Preecha Yupapin: conceptualization, supervision, review, editing and submission. All authors have read through the manuscript.

\section{References}

1. Zahid, M., N.; Jiang, J.; Rizvi, S. Reflectrometric and interferometric fiber optic sensor's principles and applications. Frontiers of Optoelectronics, 2019, 12, 215-226.

2. Musa, S.M.A.; Baharin, N.F.; Azmi, A.I.; Ibrahim, R.K.R.; Abdullah, A.S.; Noor, M.Y.M.; Qi. H. Double-clad fiber Michelson interferometer for measurement of temperature and refractive index. Microwave and Optical Technology Letters, 2018, 60, 4, 822827.

3. Jin, X.; Ou, J.; Hao, R.; Zhou, Feng.; Zhang, X.; Zheng, S.; Chi, H. Novel demodulation method for fiber-optic interferometers based on $\pi / 2$ phase modulation. IEEE Photonics Technology Letters, 2012, 24, 22, 1981-1983.

4. Benleumli, A.; Boubekeur, N.; Massicotte, D. A highly sensitivite substrate integrated waveguide interferometer applied to humidity sensing. IEEE Microwave and Wireless Components Letters, 2019, 29, 1, 68-70.

5. Cui, Y.; Wang, P. Auto-tuning and self -calibration of high-sensitivity radio frequency interferometers. IEEE Microwave and Wireless Components Letters, 2016, 26, 11, 957-959.

6. Li, X.; Warren-S.C.S.; Ebendorff-H. H.; Zhang, Y.; Nguyen, V. L. Optical fiber refractive index sensor with low detection limit and large dynamic range using a hybrid fiber interferometer. Journal of Lightwave Technology, 2019, 37, 13, $2954-2962$.

7. Li, X.; Linh, V.N.; Yong, Z.; Heike, E.H.; Stephen, C.W.S. High sensitivity Sagnac interferometer biosensor based on exposed core microstructured optical fiber. Sensors and Actuators B: Chemical, 2018, 269, 103-109.

8. Dash, J.N.; Jha, R. PCF modal interferometer based on macrobending for refractive index sensing. IEEE sensors Journal, 2015, 15, 9, 5291-5295.

9. Liu, L.; Xili, J.; Hailiang, C.; Jianshe, L.; Ying, G.; Song, Z.; Hongyu, L.; Shuguang, L. Highly sensitive temperatue sensor based on Sagnac interferometer using photonic crystal fiber with circular layout. Sensors and Actuators A: Physical, 2020, $314,112236$.

10. Liu, Q.; Liang, X.; Zhaoxia, W.; Lu, C.; Zerui, Z.; Jincheng, Z. High sensitivity photonic crystal fiber force sensor based on Sagnac interferometer for weighing. Optics and Laser Technology, 2020, 123, 105939.

11. Lv, F.; Chunyang, H.; Hui, D.; Zhongying, W.; Xianli, L. Magnetic field sensor based on micro-fiber Sagnac loop interferometer and ferrofluid. IEEE Photonics Technology Letters, 2015, 27, 22, 2327-2330. 
12. Mollaha, M.A.; Yousufalia, M.; Faysala, M.R.B.A.; Hasan, M.R.; Hossain, M.B.; Amiri, I.S. Highly sensitive photonic crystal fiber salinity sensor based on Sagnac interferometer. Results in Physics, 2020, 16, 103022.

13. Chen, W.; Shuqin, L.; Liwen, W.; Hui, Z.; Wenliang, L.; Shuisheng, J. Highly sensitive torsion sensor based on Sagnac interferometer using side-leakage photonic crystal fiber. IEEE Photonics Technology Letters, 2011, 23, 21, 1639-1641.

14. Zu, P.; Chi, C., C.; Yongxing, J.; Tianxun, G.; Yifan, Z.; Li, H., C.; Xinyong, D. A temperature insensitive twist sensor by using low birefringence photonic crystal fiber based Sagnac interferometer. IEEE Photonics Technology Letters, 2011, 23, 13, 920-922.

15. Gong, H.; Haifeng, S.; Sulei, Z.; Yongxing, J.; Xinyong, D. Curvature sensor based on hollow core photonic crystal fiber Sagnac interferometer. IEEE Sensors Journal, 2014, 14, 3, 777-780.

16. Feng, W.Q.; Zheng, Y.L.; Hwa, Y.T.; Jian, H.Y. The pore water pressure sensor based on Sagnac interferometer with polarization maintaining photonic crystal fiber for the geotechnical engineering. Measurement, 2016, 90, 208-214.

17. Sun, L.P.; Jie, L.; Long. J.; Yang. R.; Bai-Ou. G. High birefringence microfiber Sagnac interferometer based humidity sensor. Sensors and Actuators B: Chemical, 2016, 231, 696-700.

18. An, G.; Shuguang, L.; Yinghong, A.; Haiyang, W.; Xuenan, Z. Glucose sensor realized with photonic crystal fiber based Sagnac interferometer. Optics Communications, 2017, 405, 143-146.

19. Wu, B.; Chunliu, Z.; Ben, X.; Yina, L. Optical fiber hydrogen sensor with single Sagnac interferometer loop based on Vernier effect. Sensors and Actuators B: Chemical, 2018, 255, 3, 3011-3016.

20. Uomwech, K.; Sarapat, K.; Yupapin, P. P. Dynamic modulated Gaussian pulse propagation within the double panda ring resonator system. Microwave and Optical Technology Letters, 2010, 52, 8, 1818-1821.

21. Youplao, P.; Pongwongtragull, P.; Mitatha, S.; Yupapin, P. P. Crosstalk effects of quantum key distribution via a quantum router. Microwave and Optical Technology Letters, 2011, 53, 5, 1094-1099.

22. Garhwal, A.; Ray, K.; Arumona, A.E.; Bharti, G.K.; Amiri, I.S.; Yupapin, P. Spin-wave generation using MZI embedded plasmonic antennas for quantum communications. Optical and Quantum Electronics, 2020, 52:241, 1-12.

23. Arumona, A.E.; Amiri, I.S.; Punthawanunt, S.; Ray, K.; Singh, G.; Bharati, G.K.; Yupapin, P. 3D-quantum interferometer using silicon microring-embedded gold grating circuit. Microscopy Research and Technique, 2020, 83, 10, 1217-1224.

24. Prateep, P.; Surasak, C.; Yupapin, P. P. Analytical and simulation of a triple micro whispering gallery mode probe system for a 3D blood flow rate sensor. Applied Optics, 2016, 55, 33, 9504-9513.

25. Babar, S.; Weaver, J. H. Optical constants of Cu, Ag and Au revisited. Applied Optics, 2015, 54, 3, 477-481.

26. Balanis, C.A. Antenna theory analysis and design. 4th ed., Wiley, Hoboken, 2005; 1-1104. 